XX Congreso de Ecuaciones Diferenciales y Aplicaciones

X Congreso de Matemática Aplicada

Sevilla, 24-28 septiembre 2007

(pp. 1-6)

\title{
Skew-product maps with base having closed set of periodic points.
}

\author{
$\underline{\text { Juan Luis García Guirao }}^{1}$, Miguel Ángel López \\ GUERRERO $^{2}$ \\ ${ }^{1}$ Dpto. de Matemática Aplicada y Estadística Campus de la Muralla, Universidad de Politécnica de \\ Cartagena, E-30203 Cartagena. E-mails: juan.garcia@upct.es, \\ http://www.dmae.upct.es/ jlguirao. \\ ${ }^{2}$ Dpto. de Matemáticas Universidad de Castilla-La Mancha, E.U. Politécnica de Cuenca, E-16071 \\ Cuenca. E-mail: mangel.lopez@uclm.es.
}

Key words: discrete dynamical system; skew-product map; topological entropy; Li-Yorke chaos; non-wandering points

\begin{abstract}
In [Proc. ECIT-89, World Scientific, (1991), 177-183], A. N. Sharkovskiü and S. F. Kolyada stated the problem of characterization skew-product maps having zero topological entropy. It is known that, even under some additional assumptions, this aim has not been reached. In [J. Math. Anal. Appl., 287, (2003), 516-521], J. L. G. Guirao and J. Chudziak partially solved the problem in the class of skew-product maps with base map having closed set of periodic points. The present paper has two aims for this class of maps, on one hand to improve that solution showing the equivalence between the property "to have zero topological entropy" and the fact "not to be Li-Yorke chaotic in the union of the $\omega$-limit sets of recurrent points". On other hand, we show that the properties "to have closed set of periodic points" and "all nonwandering points are periodic" are not mutually equivalent properties, for doing this we disprove a result from Efremova of 1990.
\end{abstract}

\section{Introduction, Notation and Statement of the main results}

Our frame of working will be discrete dynamical systems induced by skew-product maps defined on the unit square $I^{2}=[0,1] \times[0,1]$, i.e., continuous transformations from $I^{2}$ into itself of the form $F:(x, y) \rightarrow(f(x), g(x, y))$. The maps $f$ and $g$ are respectively called the base and the fiber map of $F$. Obviously, for every $x \in I$, the maps $g_{x}$ defined by 
$g_{x}(y):=g(x, y)$ form a system of one-dimensional maps depending continuously on $x$. For having more information on this type of systems, see for instance [1], [3], [4], [14] or [15].

By $\mathcal{C}_{\Delta}\left(I^{2}\right)$ we denote the class of skew-product maps on the unit square. Let $F$ be an element of $\mathcal{C}_{\Delta}\left(I^{2}\right)$ : for every $x \in I^{2}$ and every integer $n \geq 1$, we define $F^{n}(x)=$ $F\left(F^{n-1}(x)\right)$ and $F^{0}$ as the identity map on $I^{2}$. A point $x \in I^{2}$ is said to be periodic by $F$ if there exists a positive integer $n$ such that $F^{n}(x)=x$. The smallest of the values $n$ satisfying the previous condition is called the period of $x$. By $\mathrm{P}(F)$ we denote the set of all periodic points by $F$ and by $\operatorname{Per}(F)$ we denote the set of all periods of the points of $\mathrm{P}(F)$. For an $x \in I^{2}$, we define the $\omega$-limit set $\omega_{F}(x)$ of $x$ by $F$ as the set of all $y \in I^{2}$ such that there exists a sequence of positive integers $\left\{n_{k}\right\}_{k=0}^{\infty}$ holding $F^{n_{k}}(x) \rightarrow y$, where $k \rightarrow \infty$. Let $\operatorname{Rec}(F)$ be the set of recurrent points of $F$, i.e., the set of all $x \in I^{2}$ such that $x$ is an accumulation point of $\left(F^{n}(x)\right)_{n=0}^{\infty}$. A closed invariant set $M \subseteq I^{2}$ (i.e., $F(M) \subseteq M)$ is called minimal by $F$ if it is non-empty and it does not contain proper closed invarinat subsets. By $\operatorname{UR}(F)$ we denote the set of uniformly recurrent points of $F$, i.e., all recurrent points with minimal $\omega$-limit sets. A pair of points $\{x, y\} \subset I^{2}$ is said to be a $L i$-Yorke pair of a map $F$, if simultaneously holds $\liminf _{n \rightarrow \infty} d\left(F^{n}(x), F^{n}(y)\right)=0$ and $\limsup _{n \rightarrow \infty} d\left(F^{n}(x), F^{n}(y)\right)>0$. A point $x \in I^{2}$ is a nonwandering point of $F$ provided that for any neighborhood $\mathcal{U}$ of $x$ there exists a positive integer $m$ such that $F^{m}(\mathcal{U}) \cap \mathcal{U} \neq \emptyset$. The set of nonwandering points of $F$ is denoted by $\Omega(F)$. Given a subset $A \subseteq I^{2}$, we say that $\left.F\right|_{A}$ is chaotic (in the sense of Li-Yorke, see [17]) if $A$ contains a Li-Yorke pair of $F$.

To detect the presence of simple dynamic in a given discrete system is an important problem in mathematics. The Bowen's definition (see [5]) of the notion of topological entropy is a good tool for reaching this aim. In this setting, if the system has zero topological entropy the dynamical behaviour can be understood as simple. On the contrary if the entropy is positive a complex dynamics appear. Therefore, a natural problem arrives: to find topological characterizations of the notion of zero topological entropy. For interval systems, i.e., discrete systems of the form $(I, f)$, where $f$ is a continuous self-map of $I$, there exists a long list of equivalent properties to $(P 1)$ : $f$ has zero entropy $(\mathrm{h}(f)=0)$ (see [19]). Some of the most representative of such properties are the following:

$(P 2)$ : the topological entropy of $f_{\mid \operatorname{Rec}(f)}$ is $0\left(h\left(f_{\mid \operatorname{Rec}(f)}\right)=0\right)$,

$(P 3): f_{\mid \operatorname{Rec}(f)}$ is non-chaotic,

$(P 4)$ : every recurrent point of $f$ is uniformly recurrent $(\operatorname{Rec}(f)=\mathrm{UR}(f))$,

$(P 5)$ : the period of every periodic point is power of two.

The equivalence between $(P 1)$ - $(P 5)$ for the interval case establishes an useful procedure for discover dynamical simplicity.

In 1989, A.N. Sharkovskii and S.F. Kolyada (see [18]) formulated the problem of studying the relations between the properties $(P 1)-(P 5)$ on the setting of skew-product maps of the unit square. It is well known that they are not mutually equivalent (see [2], [8], [12], [14], [16]). Moreover, even under some additional assumptions on the skew-product map $F$, the equivalence is not reached. In [13], Z. Kočan proved that in the case of skew-product maps non-decreasing on the fibres (i.e., on sets of the form $\left.I_{x}=\{x\} \times I, x \in I\right)$ conditions $(P 1),(P 2)$ and $(P 5)$ are equivalent, $(P 3)$ implies $(P 4)$ and $(P 4)$ implies $(P 1)$. However (see [13, cf. Lemma 4.2]) there exists an example of a skew-product map non-decreasing on the fibres holding $(P 2)$ but nor $(P 3)$ neither $(P 4)$ (this example is based on the ideas from [9]). The implication from $(P 4)$ to $(P 3)$ has been recently disproved by J. Chudziak et al. in [7] by taking an appropriate Floyd-Auslander minimal system and then taking 
its appropriate continuous extension to a skew-product map of the square non-decreasing on the fibres.

In [11], J.L.G. Guirao and J. Chudziak considered the problem of the equivalence of $(P 1)-(P 5)$ in the class of skew-product maps with base map having closed set of periodic points. Under such assumption was proved that conditions $(P 1)-(P 5)$ are mutually equivalent. In that setting the relation between $(P 1)$ and $(P 3)$ states that the property of having zero topological entropy is equivalent to the presence of some "order" (in the sense that there is no chaos) in the set of recurrent points for maps in $\mathcal{C}_{\Delta}\left(I^{2}\right)$ having bases with closed sets of periodic points. The first objective of the present paper is to remark that the previous result can be easily improve by proving the equivalence between $(P 1)$ and a stronger property than $(P 3)$, in the sense that we will have no chaos in a potentially bigger set than $\operatorname{Rec}(F)$, we called this new property $(S P 3)$ (strong $(P 3)$ ).

On other hand, the class of skew-product maps having base map with a closed set of periodic point, which works for solving the problem stated by Sharkovskiı and Kolyada, was studied by L. Efremova [6] in the nineties of the last century. One of the main results in the dynamics of these maps is the following theorem.

Theorem 1 (Efremova) Let $F \in \mathcal{C}_{\Delta}\left(I^{2}\right)$ with base map $f$ such that $\mathrm{P}(f)$ is closed. Then

$$
\Omega(F)=\overline{\bigcup_{x \in \mathrm{P}(f)}\{x\} \times \Omega\left(\left.F^{p_{x}}\right|_{I_{x}}\right)}
$$

where $p_{x}$ is the period of $x$.

This result has some important implications, one of them is the equivalence between the following two properties:

- $\mathrm{P}(F)$ is closed,

- $\mathrm{P}(F)=\Omega(F)$.

The second a central aim of this paper is to show that the previous equivalence does not hold by disproving Theorem 1 .

The statement of our main results on the two problems is:

Theorem A. Let $F \in \mathcal{C}_{\Delta}\left(I^{2}\right)$ with base map $f$ such that $\mathrm{P}(f)$ is closed. Then the following properties are equivalent:

(P1) F has zero topological entropy,

(SP3) F restricted to $\bigcup_{(x, y) \in \operatorname{Rec}(F)} \omega_{F}(x, y)$ is non-chaotic.

Theorem B. There exists $G \in \mathcal{C}_{\Delta}\left(I^{2}\right)$ with base map $g$ such that $\mathrm{P}(g)$ is closed, holding

$$
\Omega(G) \neq \overline{\bigcup_{x \in \mathrm{P}(g)}\{x\} \times \Omega\left(\left.G^{p_{x}}\right|_{I_{x}}\right)} .
$$




\section{Proof of the main results}

Proof of Theorem A.

On one hand, by definition (SP3) implies $(P 3)$ and by [11], $(P 3)$ implies $(P 1)$.

Let $h(F)=0$. By [10], $\operatorname{Rec}(F)=\mathrm{UR}(F)$, and thus

$$
\bigcup_{x \in \operatorname{Rec}(F)} \omega_{F}(x)=\bigcup_{x \in \operatorname{UR}(F)} \omega_{F}(x)=\mathrm{UR}(F) .
$$

Indeed, by definition every uniformly recurrent point belongs to its own $\omega$-limit set and therefore to $\bigcup_{x \in \operatorname{UR}(F)} \omega_{F}(x)$. On the other hand, let $y \in \bigcup_{x \in \mathrm{UR}(F)} \omega_{F}(x)$, so there exists $x_{0} \in \operatorname{UR}(F)$ that $y \in \omega_{F}\left(x_{0}\right)$.

By definition follows that $\omega_{F}(y) \subset \omega_{F}\left(x_{0}\right)$ and since $x$ is uniformly recurrent $\omega_{F}\left(x_{0}\right)$ is a minimal set and therefore $\omega_{F}(y)=\omega_{F}\left(x_{0}\right)$ from which we have that $y$ is a uniformly recurrent point.

Thus, $\left.F\right|_{\bigcup_{x \in \operatorname{Rec}(F)}} \omega_{F}(x)=\left.F\right|_{\operatorname{Rec}(F)}$ and it is non-chaotic, by [10].

Proof of Theorem B. As a base map let $g$ be a continuous interval map having $\mathrm{P}(g)$ closed with an attracting fixed point $y^{*}$ isolated in the set $\mathrm{P}(g)$.

For constructing the fiber map, let $h$ be a continuous interval map with a no nonwandering point $z^{*}$ but, such that a small modification on the definition of $h(z)$ becames $z^{*}$ on a nonwandering point of the modified map. Indeed, for example we consider as a map $h$ the piecewise linear map such that $0 \rightarrow \frac{7}{8}, \frac{1}{4} \rightarrow \frac{3}{8}, \frac{1}{2} \rightarrow \frac{1}{2}, \frac{3}{4} \rightarrow \frac{7}{8}$ and $1 \rightarrow 1$. Obviously, $z^{*}=\frac{1}{4}$ does not belong to $\Omega(h)$. On other hand, if we consider $h_{\delta}$ (with $\delta<\frac{1}{16}$ ) the piecewise linear map such that $h_{\delta}\left(z^{*}-\delta\right)=z^{*}-\delta$ and $h_{\delta}(z)=h(z)$ for any $z \in I \backslash\left(z^{*}-\delta\right)$, the point $z^{*} \in \Omega\left(h_{\delta}\right)$.

Since the fixed point $y^{*}$ is attracting, there exists an open neighborhood $\mathcal{V}$ of $y^{*}$ such that $\operatorname{diam}(\mathcal{V})<\frac{1}{16}$. We define a skew-product map $G: \mathcal{V} \times I \rightarrow \mathcal{V} \times I$ of the form $G(y, z)=\left(g(y), h_{\delta}(z)\right)$ where $\delta=\left|y-y^{*}\right|$. By [10] this map can be extended continuously to the whole square $I^{2}$ remaining the skew-product morphology.

Let $m^{*}=\left(y^{*}, z^{*}\right)$. We shall show that $m^{*} \in \Omega(G) \backslash \overline{\bigcup_{x \in P(g)}\{x\} \times \Omega\left(\left.G^{p_{x}}\right|_{I_{x}}\right)}$. Indeed, $y^{*}$ is isolated in $\mathrm{P}(g)$ and $h_{y^{*}}=h$, therefore $\Omega\left(h_{y^{*}}\right)=\Omega(h)$. Since $z^{*} \notin \Omega(h)$ and the set of nonwandering points is closed, $m^{*} \notin \overline{\bigcup_{x \in P(g)}\{x\} \times \Omega\left(\left.G^{p_{x}}\right|_{I_{x}}\right)}$.

On the converse, we shall verify that $m^{*} \in \Omega(G)$. By the construction of the map $h$, for any left-side open neighborhood $\mathcal{W}$ of the fixed point $c=\frac{1}{2}$, there exists a non-negative integer $m$ such that

$$
z^{*} \in h^{m-1}(\mathcal{W})
$$

Let $\mathcal{M}=\mathcal{V}^{\prime} \times \mathcal{L} \subset I^{2}$ be an open neighborhoof of $m^{*}$ where we assume that $\mathcal{V}^{\prime}, \mathcal{L} \subset$ $I$ and $\mathcal{V}^{\prime} \subset \mathcal{V}$. We have to show that there exists a non-negative integer $l$ such that $G^{l}(\mathcal{M}) \cap \mathcal{M} \neq \emptyset$.

By definition, the point $y^{*}$ is an attracting fixed point for the base map $g$, therefore there exists $y \in \mathcal{V}^{\prime} \backslash\left\{y^{*}\right\}$ such that $g^{n}(y) \in \mathcal{V}^{\prime}$ for each non-negative integer $n$. Thus, for ending the proof is enough to show the existence a non-negative integer $l$ such that $z^{*} \in K_{l}$ where by $K_{l}$ we denote the projection on the second coordinate of $G^{l}\left(\mathcal{M} \cap I_{y}\right)$.

By definition of the skew-product map $G, G^{l}\left(\mathcal{M} \cap I_{y}\right)=g\left(G\left(\mathcal{M} \cap I_{y}\right)\right)$ for every nonnegative integer $l$. Now, since $G\left(\mathcal{M} \cap I_{y}\right)$ contains certain left-side open neighborhood $\mathcal{W}$ of the fixed point $c,(3)$ ends the proof showing $m^{*} \in \Omega(G)$. 
Skew-product maps with base having closed set of periodic points

\section{References}

1. C. Arteaga, Smooth triangilar Maps of the Square with Closed Set of Periodic Points. Journal of Mathematical Analysis and Applications. 196 (1995), 987-997.

2. F. Balibrea, F. Esquembre and A. Linero, Smooth triangular Maps of type $2^{\infty}$ with Positive Topological Entropy. Inter. Journal of Bifurcation and Chaos 5 (1995), 13191324.

3. - - J.L.G. Guirao and J.I. Muñoz, Description of $\omega$-limit sets of a triangular map on $I^{2}$. Far East Journal of Dynamical Systems. 3(1) (2001), 87-101.

4. - - - - and - A Triangular map on $I^{2}$ whose $\omega$-limit Sets are all Compact Intervals of $\{0\} \times I$. Discrete and Continuous Dynamical Systems 8(4) (2002), 983-994.

5. R. Bowen, Entropy for Group Endomorphism and Homogeneous Spaces, Trans. Amer. Math. Soc. 153 (1971), 401-414.

6. L. S. Efremova, On the nonwandering set and the center of triangular maps with closed set of periodic points in the base, Dynamical Systems and Nonlinear Phenomena, Inst. Math. NAS Ukraine, Kiev, 1990, 15 - 25 (in Russian).

7. J. Chudziak, L'. Snoha and Špitalský, From a Floyd-Auslander minimal system to an odd triangular map, J. Math. Anal. Appl. 296 (2004), 316-318.

8. G. L. Forti, L. Paganoni and J. Smítal, Strange Triangular Maps of the Square, Bull. Austral. Math. Soc. 51 (1995), 395-415.

9. —- - - and —, Dynamics of Homeomorphisms on Minimal sets Generated by Triangular mappings, Bull. Austral. Math. Soc. 59 (1999), 1-20.

10. M. Grinc and L. Snoha, Jungck theorem for triangular maps and related results, Applied General Topology. 1 (2000), 83-92.

11. J.L.G. Guirao and J. Chudziak, A characterization of zero topological entropy for a class of triangular mappings, J. Math. Anal.Appl. 287 (2003), 516-521.

12. Z. Kočan, The Problem of Classification of Triangular maps with Zero Topological Entropy, Annales Mathematicae Silesianae, 13 (1999), 181-192.

13. — - Triangular maps Non-Decreasing on the Fibres, Preprint Series of Silesian University at Opava. Mathematical Institute at Opava. Preprint MA 31/2001.

14. S.F. Kolyada. On Dynamics of Triangular Maps of the Square, Ergodic Theory and Dynamical Systems. 12 (1992) 749-768.

15. — and L'. Snoha, On w-limit Sets of Triangular Maps, Real Analysis Exchange. 18(1) (1992/93), 115-130.

16. — - M. Misiurewicz and L. Snoha, Topological Entropy of Nonautonomous Piecewise Monotone Dynamical Systems on the Interval, Fundam. Math. 160, No.2 (1999), $161-181$ 
17. T. Y. Li and J. A. Yorke, Period Three implies Chaos, Amer. Math. Monthly. 82 (1975), 985-992.

18. A.N. Sharkovskiı̌ and S.F. Kolyada, On Topological Dynamics of Triangular Maps of the Plane, Proceedings of ECIT-89, Batschums, Austria, World Scientific, Singapore (1991), 177-183.

19. — - - A.G. Sivak and V.V. Fedorenko, Dynamics of One Dimensional Maps, (1997) Kluwer Academic Publishers. 